\title{
Non-contrast MRI methods as a tool for the pre-operative assessment and surveillance of the arterio-venous fistula for haemodialysis
}

\author{
Conor J. MacDonald ${ }^{1}$ - Stephen Gandy ${ }^{2}$ Eilidh C. M. Avison ${ }^{1} \cdot$ Shona Matthew ${ }^{1} \cdot$ Rose Ross $^{3} \cdot$ John G. Houston ${ }^{1,4}$
}

Received: 25 March 2018 / Revised: 22 July 2018 / Accepted: 13 August 2018 / Published online: 21 August 2018

(c) The Author(s) 2018

\begin{abstract}
Objective To compare non-contrast enhanced MRI with ultrasound (US) for measurement of arm blood vessel geometries and flow velocities in volunteers and patients with end-stage renal disease.

Materials and methods Subjects were scanned using US (reference standard), and MRI 2D time-of-flight (ToF), 2D phase contrast (PC), and 3D multi-echo data image combination (MEDIC). Patients were also scanned after arteriovenous fistula (AVF) surgery.

Results For mean vessel diameters (radial and brachial arteries; cephalic vein) MEDIC measurements were similar to US $(p>0.05)$. However, ToF underestimated the mean diameter of the cephalic vein relative to US $(p<0.05)$. For arterial velocity measurements, the mean values derived by PC-MR and US were similar $(p>0.05)$. Post-operatively, the intra-luminal signal intensity was hypo-intense at the anastomosis site using ToF and MEDIC. At the same site the outer boundary of the vessel was consistently lost on ToF, but remained clearly delineated on the MEDIC images.

Discussion With the exception of ToF, the MRI data demonstrated excellent agreement with US for measurements of vessel geometry and flow velocity. Further, the ability to clearly delineate the post-surgery vessel edges with MEDIC MRI suggests that the technique may be useful for surveillance after AVF creation or for patient-specific modelling studies.
\end{abstract}

Keywords MRI · Ultrasound · Arterio-venous fistula $\cdot$ Humans

\section{Introduction}

End-stage renal disease (ESRD) affects an estimated two million people worldwide and is increasing at a rate of around 3\% per year [1]. Patients with ESRD require some form of renal replacement therapy (RRT). Haemodialysis (HD) is the most common form of RRT; however, the provision of optimal vascular access, essential for successful $\mathrm{HD}$, has long remained a challenge. The arteriovenous fistula (AVF) first described by Brescia and Cimino in 1966 [2], revolutionised vascular access, allowing repeated access to high volume blood flow by forming a surgical anastomosis

Conor J. MacDonald

conorj.macdonald@gmail.com

1 Department of Clinical and Molecular Medicine, University of Dundee, Dundee, UK

2 Medical Physics, NHS Tayside, Dundee, UK

3 Vascular Laboratory, NHS Tayside, Dundee, UK

4 Radiology, NHS Tayside, Dundee, UK between the radial artery and cephalic vein. In response, blood flow through the venous vessel instantly increases. Ideally, the venous and arterial segments of a recently created AVF should dilate in response to the increased blood flow through the newly established low resistance pathway [3].

Unfortunately, vascular access dysfunction is a major cause of morbidity in the dialysis population-most commonly due to thrombosis, secondary to stenosis. Studies have shown that after 1 year, only around $60 \%$ of AVFs are still patent $[4,5]$ and have not required surgical intervention to remain so. Controversy exists as to the role of US in the pre-operative evaluation of the blood vessels for AVF creation. Pre-operative vessel mapping using US has been reported to reduce the immediate failure rate of AVFs and potentially increase patency rates [6-9]. However, metaanalyses by Georgiadis and Wong $[10,11]$ back up positive short-term results but fail to show an increase in long term AVF patency.

Magnetic resonance imaging (MRI) is an attractive diagnostic tool as it can provide excellent angiographic images, 
with or without the use of contrast agent $[12,13]$. Planken et al. [14] established that contrast-enhanced (CE) MRI was an accurate modality for providing pre-operative vessel diameters in patients requiring AVF creation. Further work by the same group [15] reported that CE-MRI was superior to US in detecting venous pathologies (most commonly upper arm cephalic vein occlusions) in 73 patients prior to AVF creation.

However, since 2006 there has been a marked decline in MRI based imaging involving patients with end stage renal disease and AVFs. This is likely due to the association between certain Gd-based contrast agents and nephrogenic systemic fibrosis (NSF), a fibrotic disease of the major organs [16]. Non-contrast enhanced MRI (NCE-MRI) methods are currently the safest option for this cohort. The gradient echo time of flight (ToF) sequence is commonly used in MR angiography, and relies on the inflow of blood to produce a hyper-intense signal. Various studies have reported that this sequence can visualise, assess failure and locate stenosis in AVFs [13, 17-19].

Pre-operatively, very few studies have described the use of NCE-MRI to image the vessels of patients indicated for AVF creation. Menegazzo et al. (1998) [20] compared ToF imaging with venography to assess the depiction of vein diameters prior to AVF creation. ToF measurements were better correlated with subsequent surgical findings. In 2012, Bode et al. [21] used CE-MRI and a NCE balanced turbo field echo sequence to detect stenoses prior to AVF creation. The NCE-MRI detected 66\% of (non-significant) stenosis found on CE-MRI, but importantly these were not seen on previous US imaging. More recently a T2* gradient echo "multi-echo data image combination" (MEDIC) sequence [22] has been used to highlight lower limb vessels, indicating possible future uses for this in pre-surgical mapping or surveillance of AVFs. The MEDIC sequence is a flow compensated 3D T2* weighted GRE technique where multiple echoes are acquired during the TR period via frequency encoding gradient reversals. The images acquired from each echo are then combined to form a "combination image"benefitting from improved SNR. For non-contrast measurement of blood flow velocity, the widely used technique of phase contrast MRA (PC-MRA) is available [23] — providing the opportunity for pre-operative MR assessment of vessel structure and blood flow velocity to be used within a single modality.

The purpose of this pilot study therefore was to compare 3T NCE-MRI methods against the reference standard of US for the assessment of vessel geometry and blood flow velocity in the arms of both patients and healthy volunteers at a single time-point, and also in patient volunteers before and after AVF creation. Specifically, the objective was to examine the performance of three (non contrast) MRI pulse sequences (namely MEDIC, ToF and PC MRI) for these purposes, in order to establish whether they may prove useful the pre and post-surgical assessment of patients listed for AVF creation.

\section{Materials and methods}

\section{Study cohort}

Sixteen fully consenting participants (Table 1) were recruited into the study: ten healthy volunteers (HV1-10) and six patients with end-stage renal disease (PRF) previously referred for AVF creation surgery. Three of the PRF participants were indicated for brachio-cephalic AVF creation (PRF1, PRF3, PRF5) and three were indicated for radiocephalic AVF creation (PRF2, PRF4, PRF6). All participants underwent pre-operative vessel mapping using ultrasound (S200, Siemens, USA), performed by an experienced operator, and magnetic resonance imaging (3T Siemens Magneton Trio-PrismaFIT, Erlagen, Germany) performed by an experienced clinical research team. Pre-operative images from both modalities were obtained on the same day. Post AVF surgery, all PRF participants underwent ultrasound surveillance imaging at 6 weeks. Four of the PRF participants (PRF1, PRF2, PRF3, PRF6) also underwent a single MRI surveillance session, 17-26 days after surgery.

\section{Ultrasound examinations}

For the US examinations all participants were seated with a pillow resting on their lap. The arm under investigation was extended palm up on the pillow. A tourniquet was applied to the upper arm for venous measurements and released before arterial measurements. Diameter measurements of the vessels were taken using electronic callipers. Peak systolic velocity (PSV) values were measured as the highest single point on the Doppler reading with an accuracy of $0.01 \mathrm{~m} / \mathrm{s}$. The velocity values obtained were subsequently used as a guide to assist with identifying a suitable velocity encoding (VENC) value on the subsequent phase contrast MRA sequences.

Table 1 Study participant demographics

\begin{tabular}{ll}
\hline Characteristic & Number $(N=16)$ \\
\hline Age (years) & $44 \pm 16$ \\
Caucasian & 16 \\
Diabetes mellitus & 4 \\
Atrial fibrillation & 2 \\
Heart failure & 1 \\
Upper arm imaging & 5 \\
Lower arm imaging & 11 \\
\hline
\end{tabular}




\section{Pre-operative assessment}

Lower arm evaluation The cephalic vein was identified at its most distal segment, and diameter measurements taken at this location. The diameter of the radial artery was measured at the wrist position. An assessment was made of vessel uniformity and the presence of multiple branches was noted, if present. The artery was then assessed in a longitudinal view using spectral Doppler to record the peak systolic velocity (PSV) and phasicity (presence of reverse velocity component and a clear diastolic peak velocity component).

Upper arm evaluation The cephalic vein and brachial artery were identified at the cubital fossa and their diameters were measured at this location. Vessel uniformity and branches were assessed as before. As with the radial artery, the PSV was measured using a longitudinal view. An oil capsule was then placed on the skin surface at the proposed location of the surgical anastomosis in order to guide MRI imagingi.e., to ensure that the same anatomic areas were observed using both imaging modalities.

\section{Post-operative assessment}

The vein was identified central to the anastomosis. Diameter and velocity measurements were taken $6 \mathrm{~cm}$ proximal to the anastomosis, and this process was also repeated for the artery. An oil capsule was then placed on the skin surface at the location of the surgical anastomosis in order to guide MRI imaging.

\section{MR imaging}

All participants were placed head first and supine into the scanner bore with their arms relaxed by their side. An 8-channel "small flex" RF coil was placed around the arm region of interest. The participant was positioned slightly off-axis in relation to the scanner bore, in order to ensure that the arm (anatomical area of interest) was as near as possible to the isocentre of the magnetic field.

\section{Morphology assessment}

A 2D gradient echo localiser sequence was used for initial visualisation of the proposed anastomosis area. Following this, a 2D time-of-flight (TOF) MR sequence was applied in an axial oblique orientation at either the upper or lower arm (see Table 1) perpendicular to the long bones, and region coverage was maintained at approximately $10-15 \mathrm{~cm}$. Imaging parameters used were repetition time (TR) and echo time (TE) of $14 \mathrm{~ms}$ and $5.8 \mathrm{~ms}$, respectively, flip angle (FA) of $18^{\circ}$, and a $1.5 \mathrm{~mm}$ slice thickness (ST). Images were acquired using a single average over a field of view (FOV) of $140 \mathrm{~mm}$, with an imaging matrix of $512 \times 512$ pixels (no interpolation), and receiver bandwidth of $165 \mathrm{~Hz} /$ pixel. This was followed by a 3D T2* multi-echo data image combination (MEDIC) sequence (TR/TE: 29/16 ms, FA: 30, slice thickness: $1.06 \mathrm{~mm}$ (176 slices in the imaging block), FoV: $136 \mathrm{~mm}$, matrix: $512 \times 512$ pixels (no interpolation), and receiver bandwidth: $160 \mathrm{~Hz} /$ pixel) over the same area.

\section{Velocity assessment}

Through plane blood velocity measurements were obtained in the artery and vein of interest at distal and proximal positions relative to the bifurcation or anastomosis site. A retrospectively ECG gated 2D phase contrast MRA sequence (PC-MRA) was used to derive velocity measurements (TR/TE: 99.7/7.62 ms, FA: 20 , FOV $100 \mathrm{~mm}$, matrix $192 \times 115$ pixels, receiver bandwidth: $440 \mathrm{~Hz} /$ pixel, VENC: $10-250 \mathrm{~cm} / \mathrm{s}$ (depending on whether artery or vein) and 16-64 temporal phases over the cardiac cycle).

\section{Image analysis}

The MEDIC, ToF and PC-MRI sequences were exported in DICOM format to an offline computer. Osirix Lite (Pixemo, Switzerland) was used as a database, and for diameter and area measurements. Transverse image slices from the MEDIC and ToF sequences were used for region-of-interest (ROI) analysis of vessel diameter and area. Diameter measurements were made of the vessel diameter in the major and minor axis for each image slice using electronic calipers in Osirix, and vessel area was plotted as a function of distance from the anastomosis or bifurcation. Anatomical landmarks (e.g., bifurcations of other vessels) were used to register adjacent measurements between the two time-points. Trend lines were plotted using a 3-point average.

Semi-automatic segmentation and analysis of the PCMRI images was undertaken using Segment (Medviso, Sweden). A ROI was drawn around the vessel of interest and automatic propagation of the ROI was processed through all images in the series. Flow and velocity information of blood in the vessel ROI was extracted. For comparison with US, the diameters measured by MRI were averaged over a $1 \mathrm{~cm}$ region approximately $6 \mathrm{~cm}$ from the anastomosis or oil capsule-i.e., consistent with the US imaging locations.

\section{Statistical analysis}

All statistical analysis was conducted using RStudio (RStudio Inc, US). All datasets were assessed for normality using the Shapiro-Wilks Normality test. Statistical comparison of means was subsequently performed using a student's $t$ test for normally distributed data, or the Mann-Whitney test for non-normal data. Paired equivalents were used when 
appropriate. Bland-Altman style plots were used to visualise agreement between the modalities. Graphs were plotted using Python with the "matplotlib" and "Seaborn" packages.

\section{Compliance with ethical standards}

The study received ethical approval from the East of Scotland Research and Ethics Service (EoSRES). Written informed consent was obtained from all individual participants included in the study.

\section{Results}

\section{Imaging and surgical outcomes}

All 16 participants were scanned successfully using both US and MRI, although initial observation of the images revealed that the following exclusions were required: ToF: one dataset (PRF5)—unreadable due to participant movement; MEDIC: one dataset (HV4) - unreadable due to participant movement; PC-MRI: two datasets (PRF4, HV2)—arterial VENC sub-optimal; US: one dataset (HV6) - file data corrupted during storage. Figure 1 shows example axial image slices acquired at the radio-cephalic region of the upper arm of a healthy volunteer using (i) $\mathrm{T} 2 *$ MEDIC and (ii) $2 \mathrm{D} \mathrm{ToF}$ MRA.

Surgically, AVF creation was initially successful for all but one participant (PRF1). In this unsuccessful case it was necessary to abandon the procedure due to calcification of the vessels, although a second procedure was subsequently successful at a more proximal site. Participant PRF6 developed a post-surgical infection at the anastomosis site, but with no long-term complications.

\section{Morphology results: 2D TOF vs. MEDIC vs. US diameter comparisons}

When data acquired using ToF, MEDIC and US were compared (Fig. 2), mean arterial diameters were found to be consistent across all imaging modalities $(3.2 \pm 0.7 \mathrm{~mm}$ for ToF; $3.4 \pm 0.8 \mathrm{~mm}$ for MEDIC; $3.5 \pm 0.7 \mathrm{~mm}$ for US; $p>0.05$ for all). However, statistical differences were noted when comparing the mean venous diameters measured by ToF $(3.5 \pm 1.2 \mathrm{~mm})$ to those measured by both MEDIC $(4.4 \pm 1.6 \mathrm{~mm})$ and US $(4.6 \pm 1.0 \mathrm{~mm}) ; p<0.05$. Bland-Altman style plots (Fig. 3) demonstrate good agreement between MRI (both MEDIC and ToF) and US for all artery diameters, with mean values close to zero. However, a clear underestimation of venous diameters was observed for data derived from the ToF sequence.

When participants were split into PRF $(n=6)$ and HV groups $(n=10)$, mean arterial diameters were consistent when measured with US $(3.5 \pm 1.0 \mathrm{~mm}$ for PRF; $3.5 \pm 0.5 \mathrm{~mm}$ for HV). However, mean arterial diameters were lower for the PRF cohort relative to the HV cohort when derived from both NCE-MRA sequences (MEDIC: $3.0 \pm 1.0 \mathrm{~mm}$ for PRF, $3.8 \pm 0.5 \mathrm{~mm}$ for $\mathrm{HV}$ ); (ToF: $2.7 \pm 0.9 \mathrm{~mm}$ for PRF, $3.5 \pm 0.5 \mathrm{~mm}$ for $\mathrm{HV}) ; p<0.05$ for each.

For the veins, mean measured diameters were consistently smaller in the PRF cohort relative to the HV cohort, irrespective of whether US or MRI was used (US: $3.9 \pm 1.0 \mathrm{~mm}$ for PRF, $5.0 \pm 0.8 \mathrm{~mm}$ for $\mathrm{HV}$; MEDIC: $3.0 \pm 1.5 \mathrm{~mm}$ for PRF, $5.3 \pm 0.6 \mathrm{~mm}$ for $\mathrm{HV}$; ToF $2.6 \pm 0.8 \mathrm{~mm}$ for PRF, $4.0 \pm 1.0 \mathrm{~mm}$ for $\mathrm{HV}) ; p<0.05$ for all.
Fig. 1 Example axial image slices acquired at the radiocephalic region of the upper arm of a healthy volunteer using a T2* MEDIC and b 2D ToF MRA

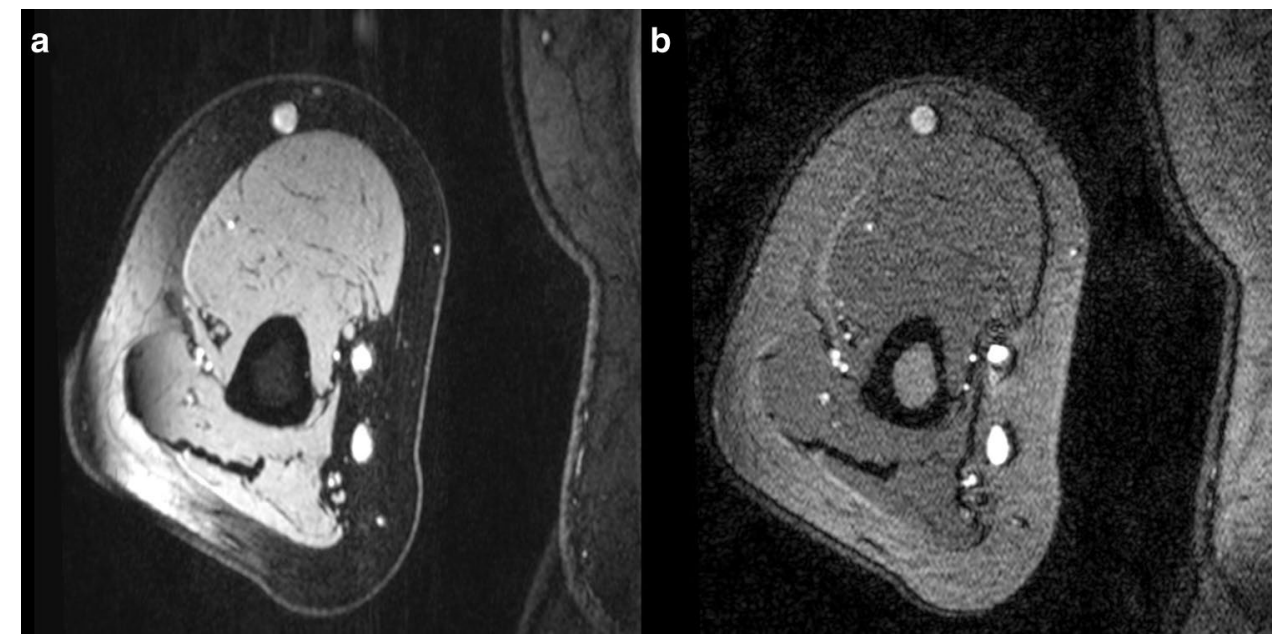


Fig. 2 Illustrations of arterial and venous diameter measurements-using ToF, MEDIC and US

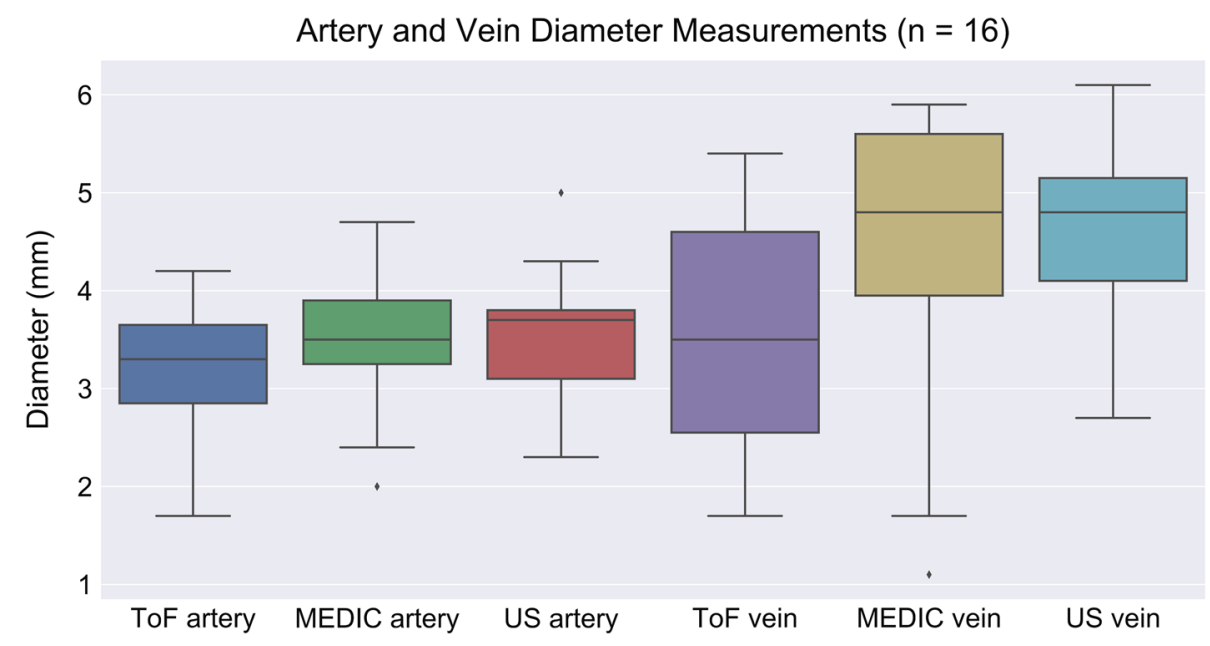

a BA-style plot for vein measurements on MEDIC \& US

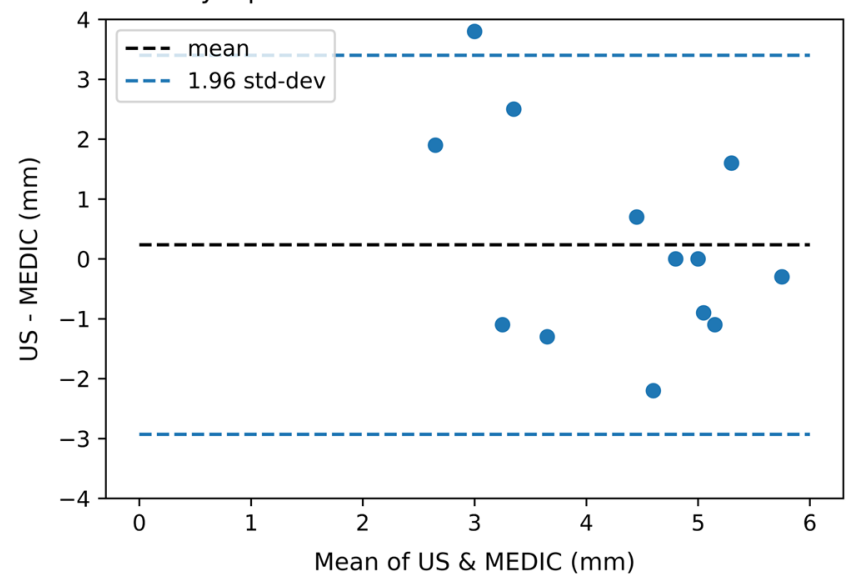

C BA-style plot for artery measurements on ToF \& US

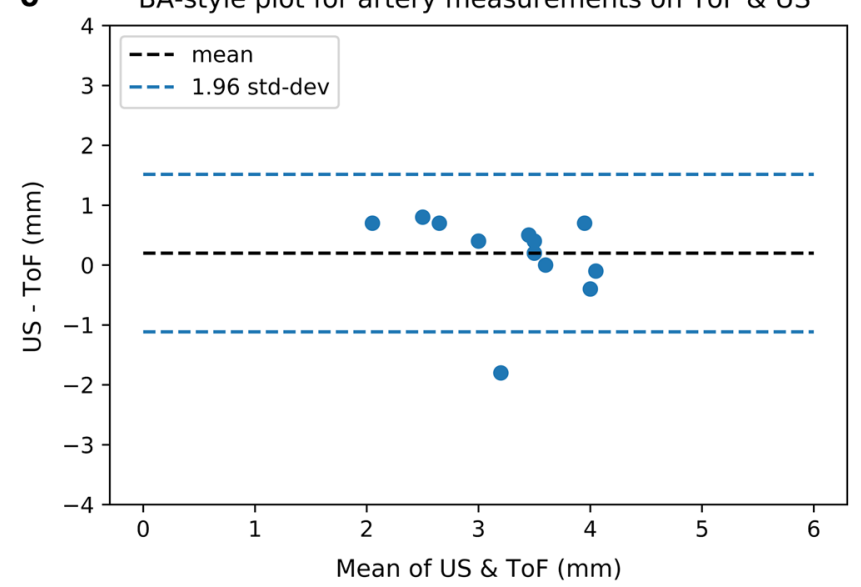

Fig. 3 Bland-Altman style plots depicting mean difference between: a artery diameter measurements from MEDIC MRI and US; b vein diameter measurements on MEDIC MRI and US; $\mathbf{c}$ artery diameter b BA-style plot for artery measurements on MEDIC \& US

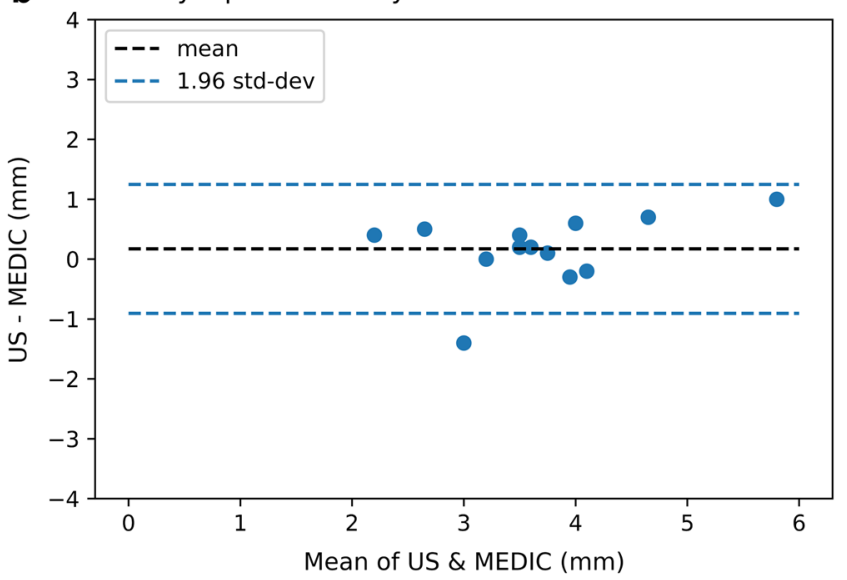

d BA-style plot for vein measurements on ToF \& US

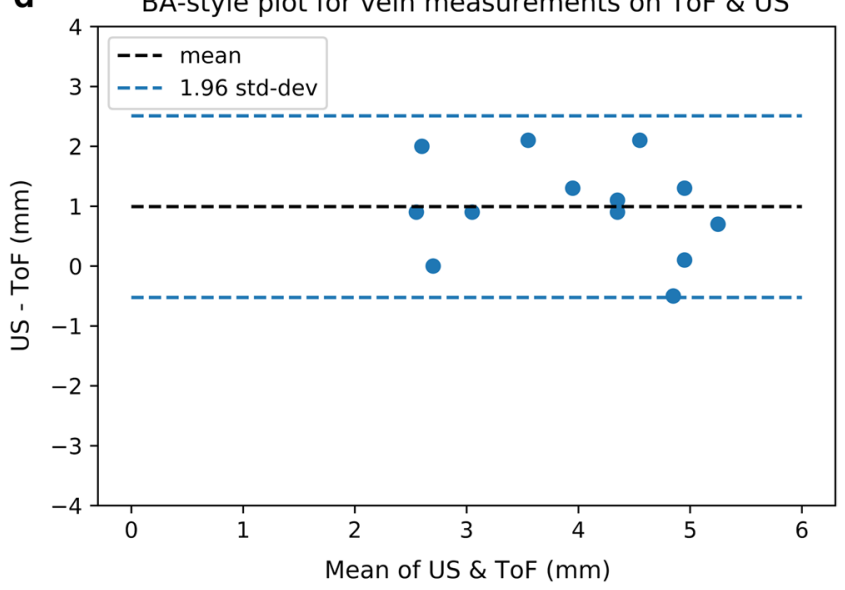

measurements on ToF MRI and US; $\mathbf{d}$ vein diameter measurements on ToF MRI and US 


\section{Flow velocity results: PC-MRA vs. US velocity comparisons}

For arterial flow velocity measurements, the mean velocities measured by MRI $(51.4 \pm 17.2 \mathrm{~cm} / \mathrm{s})$ and US $(53.2 \pm 12.6 \mathrm{~cm} / \mathrm{s})$ were similar $(p>0.05)$. When the data were considered on an individual basis, the bias between the MRI and US observations was 0.54 and the "MRI versus US" root mean square (RMS) coefficient of variation $(\mathrm{CoV})$ was $11.8 \%$.

Similarly, when the data were sub-divided into PRF and HV cohorts, the mean velocities measured by MRI and US were still consistent (PC-MRI: $49.8 \pm 25.6 \mathrm{~cm} / \mathrm{s}$ for PRF, $52.2 \pm 12 / 4 \mathrm{~cm} / \mathrm{s}$ for HV); and US: $55.0 \pm 7.7 \mathrm{~cm} / \mathrm{s}$ for PRF, $52.0 \pm 15 / 4 \mathrm{~cm} / \mathrm{s}$ for HV); $p>0.05$ for all.

\section{Pre-operative and post-operative observations of vessel morphology and flow}

Pre-operative and post-operative arterial and venous diameter measurements were measured, but statistical testing of these measurements were not attempted since the number of subjects involved was small $(n=4)$. The RMS differences between the post-surgery minus pre-surgery measured vein diameters were $3.2 \mathrm{~mm}$ (ToF), $3.9 \mathrm{~mm}$ (MEDIC) and $2.2 \mathrm{~mm}$ (US), and for the arterial diameters the RMS differences were $1.7 \mathrm{~mm}$ (ToF), $2.2 \mathrm{~mm}$ (MEDIC) and $1.3 \mathrm{~mm}$ (US). Looking in more detail at the vein morphology over the full length of the MR imaging block, all veins studied developed larger cross-sectional areas after surgery relative to their pre-surgical dimensions. However, the cross-sectional area changes were not uniform across the full length of the vein area scanned (see Fig. 4).

Arterial peak velocities were markedly increased after surgery. The RMS differences between the post-surgery minus pre-surgery measured arterial velocities were $164.3 \mathrm{~cm} / \mathrm{s}$ (PC-MR) and $183.6 \mathrm{~cm} / \mathrm{s}$ (US). Post-operatively the arterial waveforms were altered, with loss of their characteristic tri-phasic pattern (a lack of reverse velocity component and a loss of a clear diastolic peak velocity component).
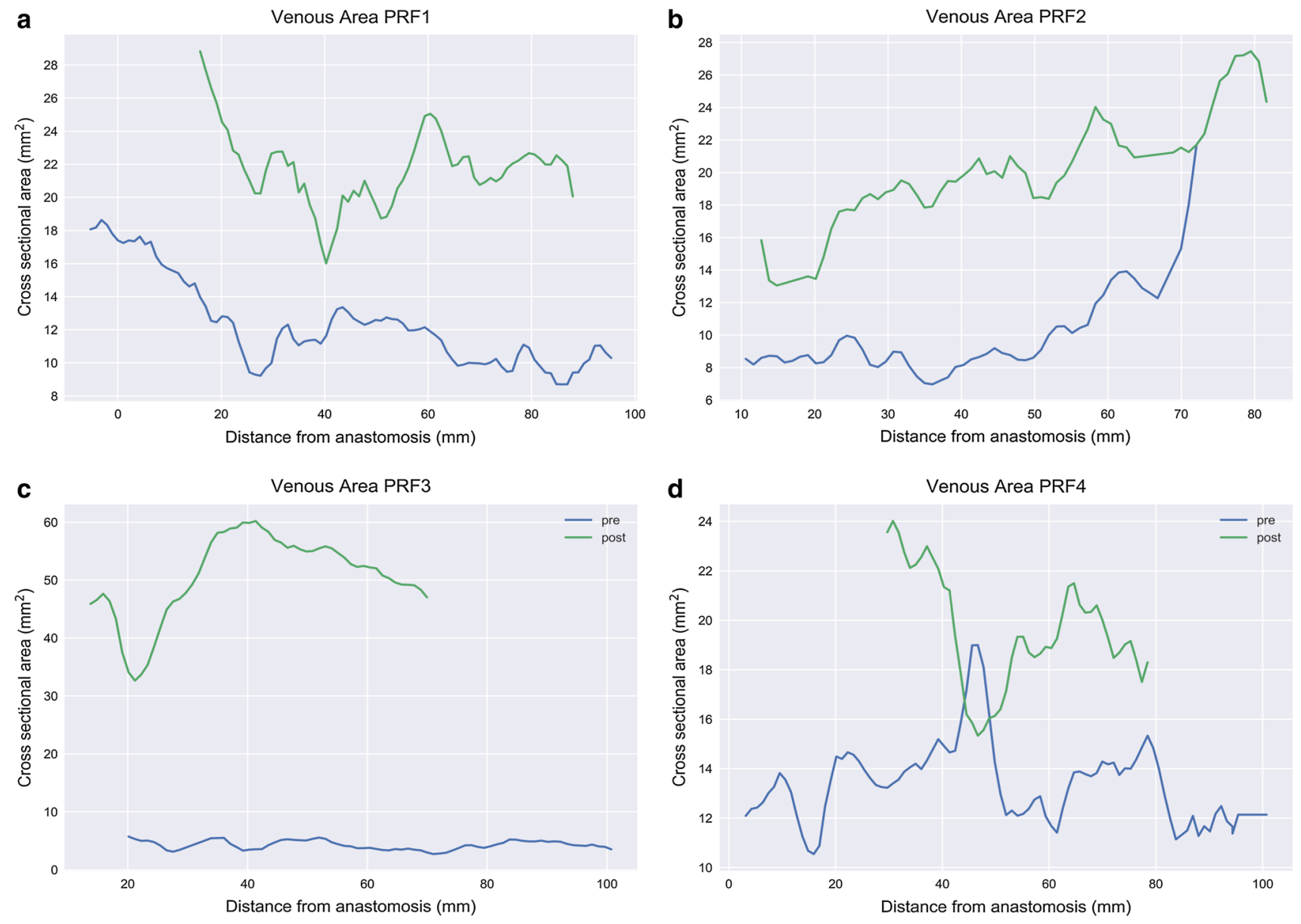

Fig. 4 Graphs depicting pre and post-operative venous area for participants a PRF1; b PRF2; $\mathbf{c}$ PRF3; d PRF4. Areas of narrowing are visible in participants PRF3 and PRF4 


\section{Imaging features of the TOF and MEDIC MR sequences for AVF visualisation}

On all pre-surgical images (ToF and MEDIC) the vessel lumen 'flow' signal intensity was hyper-intense, as expected. However, on the post-surgical images the vessel lumen was hyper-intense at locations proximal to the anastomosis but the signal was lost within the anastomosis itself. On the ToF images it was not possible to discern the vessel pathway of the anastomosis since the vessel edges were unclear, but on the MEDIC images the vessel edges remained consistently and clearly visible - thus enabling a better view of the stationary structures associated with the anastomosis. Example post-surgical MEDIC images are shown in Fig. 5.

\section{Discussion}

In this pilot study, the feasibility of using $3 \mathrm{~T}$ MRI techniques for the assessment of upper and lower arm blood flow geometries and velocities at potential AVF sites is described. For morphological assessments, the MRI T2* MEDIC technique used compared favourably against the current reference standard of US, with very similar diameters obtained in patients and healthy volunteers. Further, the "combined" observation of hypo-intense vessel lumen with hyper-intense vessel edges in the vicinity of the anastomosis using MEDIC was a consistent finding in the four examples studied. Further studies are warranted to explore this pattern of contrast behaviour, but initial evidence suggests that it may have the potential to assist with the visualisation of AVF remodelling events. Increased knowledge of the remodelling process of arteriovenous fistulae is required to help improve scientific understanding of AVF failure, and ultimately to facilitate the pre-operative identification of patients whose fistulae may fail to reach maturation. Current guidelines recommend physical examination or US, but these cannot provide the anatomical detail required to increase the knowledge of the remodelling process. The purpose of this work was to explore wider imaging opportunities as an alternative pre-operative assessment to gain extra anatomical information. We utilised two MRI sequences (ToF and MEDIC), alongside US to compare the geometries of arteries and veins in patients with renal failure $(n=6)$ and healthy volunteers $(n=10)$. In a subset of the patients, AVF remodelling was also monitored post-surgery.

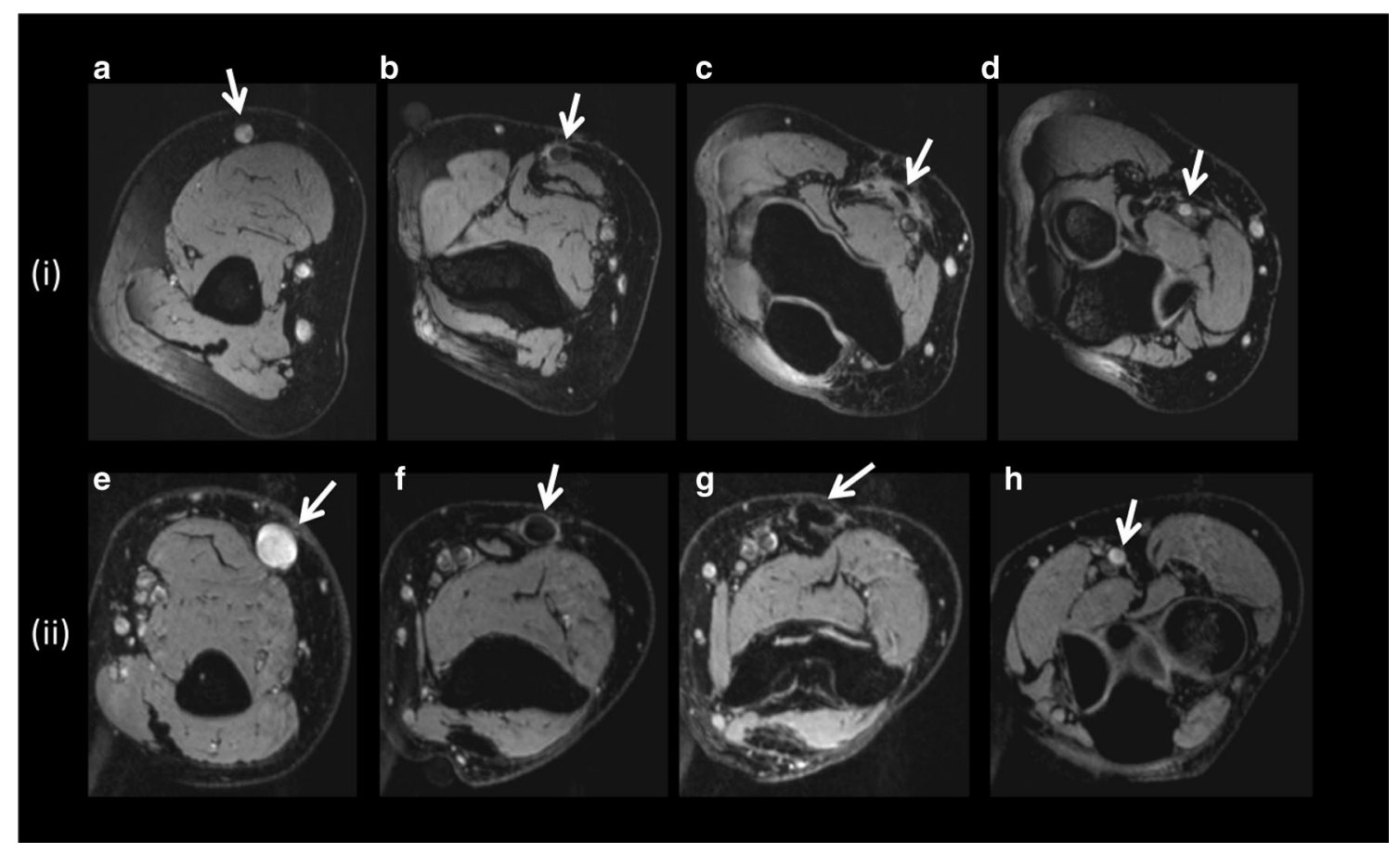

Fig. 5 Example 3T gradient-echo "MEDIC" MR images of the elbow region of two different participants, (i) and (ii), who each underwent AVF placement. (A) and (E) show examples of high luminal signal intensity in the cephalic veins at a location a few $\mathrm{cm}$ proximal to the anastomosis site. The luminal diameter is slightly enlarged in participant (i) and grossly enlarged in participant (ii); (B) and (F) show examples of hypo-intense luminal signal intensity together with hyper-intense "vessel edge" signal intensity at the approach to the anastomosis site; $(C)$ and $(G)$ each illustrate the flat "swing site" location of the anastomosis; and (D) and (H) each illustrate the hyperintense luminal signal intensity in the brachial artery distal to the anastomosis site. The images were obtained 24 days post-surgery for participant (i) and 26 days post-surgery for participant (ii) 
The MEDIC sequence was in agreement with US for all morphological measurements made across the whole group $(n=16)$. However, the ToF sequence underestimated the diameter of the cephalic vein relative to both MEDIC and US. Although the ToF sequence can be useful in detection of stenosis, these results suggest that if the depiction of accurate vessel diameter information is important, then MEDIC would be a better choice. The strong agreement seen between the MEDIC sequence and US confirms that this may be a viable modality for mapping patient vasculature prior to AVF surgery. An additional advantage is that MEDIC was able to depict both the arterial and venous system in the arm in a single session, whereas ToF was unable to depict adequately the venous vessels in some cases. Importantly, US is recognised by current Kidney Disease Outcomes Quality Initiative (KDOQI) guidelines-thus, the agreement between the two modalities also helps to provide an indirect validation of the MEDIC sequence for assessment of vessel morphology. However, it is important to state that the MEDIC sequence is unproven for conventional clinical MR angiography-and we would not recommend it for radiological stenosis assessment since formal performance studies (e.g., comparisons with DSA) have not been done. In this research study we have used MEDIC specifically for vessel diameter tracking.

The MEDIC and US morphology measures were generally in good agreement with each other. It is possible that the higher SNR of the 3D MEDIC sequence enabled clearer visualisation of the vessel diameters relative to those of the ToF images. Further, the presence of the higher signal "vessel edge" detail on MEDIC (clearly seen in the anastamosis regions on the patients) may additionally contribute to the "true vessel width" not seen on 2D-TOF. From the perspective of US, a possible concern is the fact that tourniquets are routinely used to instigate a level of venous congestion-potentially affecting the vein diameters. However, work presented by van Bemmelen et al. [24] determined that differences between US diameter measurement values obtained with and without a tourniquet were not significant for either the cephalic or the basilic vein. They concluded that vein diameters are affected by warm water immersion, but not the use of a tourniquet.

The lack of MRI contrast media used in this study completely removes any possibility of NSF or contrast induced nephropathy. The MEDIC sequence has the advantage of acquiring the imaging in 3D-thus allowing measurements to be made throughout the length of the vessel, unlike selective measurements from US. Further, the MEDIC does not appear to suffer from through-plane signal losses in quite the same way as ToF. An extension to consider the central vessels would also be feasible as part of a future study. Previous research by Planken et al. [14] has reported that contrast enhanced MR angiography (CE-MRA) is more accurate than
US when comparing diameter measurements made during AVF surgery, but no other similar study has been performed using a NCE-sequence. In our study, the radial artery diameter in the PRF participants as measured by MEDIC was in agreement with literature values measured by US [25]. Vessels used for AVF creation were above the recommended minimum vessel diameters stated in the literature $[9,26$, 27], but lower than that recommended by Lauvao et al. [28].

Our results show that, pre-operatively, the vessels can have a heterogeneous cross-sectional area (CSA). The implications of this for AVF maturation requires further study. It is possible that narrower (but non-stenotic) regions which exist pre-operatively, may be more prone to stenosis development post-AVF creation. Post-operatively, vessels had achieved or were approaching the recommended diameter of $6 \mathrm{~mm}$ for cannulation. This adds to the sentiment that pre-operative vessel diameters are not the major predictor of maturation that some studies would suggest. Heterogeneous changes in the venous segment of an AVF have been observed before $[29,30]$. In this study, we included a preoperative analysis, enabling us to determine if any areas had undergone a decrease in area. It is possible that the areas of post-operative narrowing observed could be the result of neo-intimal hyperplasia, and that the areas of lower dilation are related to local endothelial dysfunction or abnormal flow patterns. However, this would need to be determined by further studies involving larger cohorts, multiple post-surgical analyses and/or haemodynamic simulations.

Few studies have focussed on the changes that occur in the arterial segment, post AVF creation. Corpateux et al. [3] found that the radial artery did not significantly change over a 3-month period. However, we noted large increases in the CSA of the radial arteries studied. The observations noted by us may corroborate the use of MRI for providing additional anatomical data, which may form part of the larger picture of AVF-remodelling.

The purpose of this study was not to demonstrate the diagnostic ability of the MRI sequences for AVF stenosis detection, although regions of narrowing were identified in the venous segment of two AVFs. One of these participants (PRF3) did develop a stenosis at a later date, which was detected by routine US imaging. Further work is needed to determine the pattern of stenosis formation post AVF, and whether MRI may have a role in targeted post-operative stenosis screening.

MRI is rarely used in a clinical setting with for ESRD patients due to financial constraints, patient contraindications and concerns over the use of MRI contrast agents. Contraindication rates to MRI imaging of around $10 \%$ in patients with ESRD have been reported previously [12, 31]. Research involving MRI in patients on, or awaiting haemodialysis has also slowed considerably since the occurrence of NSF. Non-contrast enhanced sequences such as black-blood or 
ToF MRI have been studied previously [29, 30], and the relative limitations of each technique are reported. The MEDIC sequence used in this study (a gradient echo technique with $\mathrm{T} 2 *$ weighting) provides good agreement with US measures, but in common with ToF techniques it also suffers from inflow-related signal losses at the anastomosis. However, the MEDIC sequence does provide good vessel edge detail at the locations in and around the anastomosis, and it is possible that this may prove to be useful as a surveillance tool to characterise the development of stationary structures such as possible stenosis sites.

This study has several limitations. Firstly, detailed optimisation work was not performed on the ToF sequence to maximise the vessel/background contrast for use at the chosen anatomical location - the vendor default parameters were utilised. The flip angle was slightly lower than one might normally expect, although since we were observing relatively slow flow within the veins the use of a larger flip angle may have resulted in partial saturation of the venous signal. It is difficult to optimise the sequence when both arteries and veins are being observed, although we accept that the negative effect of using a smaller flip angle will be an elevated signal from stationary tissue. Lower flip angles were also necessary in order for the acquisition to stay within acceptable SAR limits on our 3T scanner for the chosen TR. Other experimental complexities included the fact that the anatomy was, by definition, positioned at the periphery of the scanner bore in an area of relatively heterogeneous magnetic field-leading to unavoidable regions of inhomogeneous signal intensity. Finally, we were not able to apply fat suppression because doing this would have resulted in a longer sequence TR-leading to unacceptably long scan times. The TR was also implemented with consideration of the expected venous flow velocities likely to be present in the healthy volunteers. We estimated that the slowest diastolic blood flow velocities present would be about $10 \mathrm{~cm} / \mathrm{s}$, and using an estimate that the TR would need to be equal or greater than the slice thickness $(1.5 \mathrm{~mm})$ divided by the minimum flow velocity $(10 \mathrm{~cm} / \mathrm{s})$ to allow full in-flow of unsaturated blood-i.e., the TR would need to be a minimum of approximately $15 \mathrm{~ms}$. Our choice of TR $14.5 \mathrm{~ms}$ was, therefore, "at the limit" in terms of the slowest blood flow velocities likely to be encountered. However, since the cardiac cycle was postulated to involve velocities mostly above $10 \mathrm{~cm} / \mathrm{s}$, we felt that using this short TR would be the best solution to ensure the shortest scan time-and lower chances of subject motion artefacts. We also used a TE of $5.8 \mathrm{~ms}$ (the shortest $\mathrm{TE}$ to acquire fat/water out of phase images at 3.0T), which was implemented to ensure that the signal from water and fat were out of phase-thus helping to minimise stationary background tissue signals a little further.

Other modern MRI pulse sequences are potentially available for non-contrast enhanced visualisation of the vascular system. These include, for example, "black blood" spin echo sequences (utilising double or triple inversion pulses to null the signal from the vessels), and cardiac gated gradient-echo based sequences that typically use inversion recovery along with a steady state free precession read-out after a systolic inflow period to allow movement of hyper-intense blood into the imaging plane. Common examples of these are Non-Contrast MRA of Arteries and Veins (NATIVE, Siemens), Quiescent Interval Steady State (QISS, Siemens); Balanced-Triggered Angiography Non-Contrast Enhanced (B-TRANCE, Philips); Inhance Inflow IR (IFIR, GE) and Time Spatial Labeling Inversion Pulse (TimeSLIP, Cannon). These sequences may be useful in the future when it becomes possible to optimise them easily for arteries and veins interchangeably.

In summary, our study has demonstrated that the MEDIC sequence can offer accurate depiction of both the arterial and venous dimensions in healthy volunteers and for patients requiring detailed vessel mapping of AVF sites. This information can be acquired in a single imaging session and does not rely on contrast agent injections, which makes it suitable for imaging wider patient groups with ESRD.

\section{Conclusion}

This pilot study has demonstrated that non-contrast enhanced MRI can be used to describe vessel morphology and blood flow at anatomical sites associated with AVF surgery-with results comparable to the reference standard of US. Additionally, the pattern of image contrast associated with the MEDIC sequence at the anastomosis site may assist with future studies designed to examine the AVF maturation process without the need for contrast agents.

Author contributions CM analysis and interpretation of data, drafting of manuscript. SG acquisition of data, drafting of manuscript, critical revision. SM acquisition of data, study concept and design. EA analysis and interpretation of data. RR critical revision. JGH study conception and design.

Funding This study was funded by the European Unions 7th Framework Program for research, technological development and demonstration under grant agreement no. 324487 (ReDVA).

\section{Compliance with ethical standards}

Conflict of interest The authors declare that they have no conflict of interest.

Ethical standards All procedures performed in the study were in accordance with the institutional research committee and with the 1964 Helsinki declaration and its later amendments. 
Informed consent Informed consent was obtained from all individual participants included in the study.

Open Access This article is distributed under the terms of the Creative Commons Attribution 4.0 International License (http://creativeco mmons.org/licenses/by/4.0/), which permits unrestricted use, distribution, and reproduction in any medium, provided you give appropriate credit to the original author(s) and the source, provide a link to the Creative Commons license, and indicate if changes were made.

\section{References}

1. Collins AJ, Foley RN, Gilbertson DT, Chen S (2009) The state of chronic kidney disease, ESRD, and morbidity and mortality in the 1st year of dialysis. Clin J Am Soc Nephrol 4(SUPPL. 1):5-11

2. Brescia MJ, Cimino JE, Appel K, Hurwich BJ (1966) Chronic hemodialysis using venipuncture and a surgically created arteriovenous fistula. N Engl J Med 275(20):1089-1092

3. Corpataux J, Haesler E, Silacci P, Ris HB, Hayoz D (2002) Lowpressure environment and remodelling of the forearm vein in Brescia-Cimino haemodialysis access. Nephrol Dial Transplant 17(6):1057-1062

4. Almasri J, Alsawas M, Mainou M, Mustafa RA, Wang Z, Woo K, Cull DL, Murad MH (2016) Outcomes of vascular access for hemodialysis: a systematic review and meta-analysis. J Vasc Surg 64(1):236-243

5. Rooijens PPGM, Tordoir JHM, Stijnen T, Burgmans JPJ, Yo TI (2004) Radiocephalic wrist arteriovenous fistula for hemodialysis: meta-analysis indicates a high primary failure rate. Eur J Vasc Endovasc Surg 28(6):583-589

6. Robbin ML, Gallichio MH, Deierhoi MH, Young CJ, Weber TM, Allon M (2000) US vascular mapping before hemodialysis access placement. Radiology 217(1):83-88

7. Allon M, Lockhart ME, Lilly RZ, Gallichio MH, Young CJ, Barker J, Deierhoi MH, Robbin ML (2001) Effect of preoperative sonographic mapping on vascular access outcomes in hemodialysis patients. Kidney Int 60(5):2013-2020

8. Mihmanli I, Besirli K, Kurugoglu S, Atakir K, Haider S, Ogut G, Numan F, Canturk E, Sayin AG (2001) Cephalic vein and hemodialysis fistula: surgeon's observation versus color Doppler ultrasonographic findings. J Ultrasound Med 20(3):217-222

9. Mendes RR, Farber MA, Marston WA, Dinwiddie LC, Keagy BA, Burnham SJ (2002) Prediction of wrist arteriovenous fistula maturation with preoperative vein mapping with ultrasonography. J Vasc Surg 36(3):460-463

10. Georgiadis GS, Charalampidis DG, Argyriou C, Georgakarakos EI, Lazarides MK (2015) The necessity for routine pre-operative ultrasound mapping before arteriovenous fistula creation: a metaanalysis. Eur J Vasc Endovasc Surg 49(5):600-605

11. Wong CS, McNicholas N, Healy D, Clarke-Moloney M, Coffey JC, Grace PA, Walsh SR (2013) A systematic review of preoperative duplex ultrasonography and arteriovenous fistula formation. J Vasc Surg 57(4):1129-1133

12. Doelman C, Duijm Lucien EM, Liem YS, Froger CL, Tielbeek AV, Donkers-van Rossum AB, Cuypers PWM, Douwes-Draaijer P, Buth J, van den Bosch HCM (2005) Stenosis detection in failing hemodialysis access fistulas and grafts: comparison of color Doppler ultrasonography, contrast-enhanced magnetic resonance angiography, and digital subtraction angiography. J Vasc Surg 42(4):739-746

13. Gonzalez AJ, Casey KM, Drinkwine BJ, Weiss JS (2016) Series of noncontrast time-of-flight magnetic resonance angiographies to identify problems with arteriovenous fistula maturation. Ann Vasc Surg 30:93-99

14. Planken NR, Tordoir JH, Duijm LE, van den Bosch HC, van der Sande FM, Kooman JP, de Haan MW, Leiner T (2008) Magnetic resonance angiographic assessment of upper extremity vessels prior to vascular access surgery: feasibility and accuracy. Eur Radiol 18(1):158-167

15. Planken NR, Leiner T, Nijenhuis RJ, Duijm LE, Cuypers PW, Douwes- Draaijer P, Van der Sande FM, Kessels AG, Tordoir JH (2008) Contrast-enhanced magnetic resonance angiography findings prior to hemodialysis vascular access creation: a prospective analysis. J Vasc Access 9(4):269-277

16. Larson KN, Gagnon AL, Darling MD, Patterson JW, Cropley TG (2015) Nephrogenic systemic fibrosis manifesting a decade after exposure to gadolinium. JAMA Dermatol 151(10):1117-1120

17. Harada S, Yamakido M, Yalniz MT et al (1996) Magnetic resonance angiography as a technique for the visualization of hemodialysis shunts. Nephron 73(1):73-78

18. Laissy JP, Menegazzo D, Debray MP, Loshkajian A, Viron B, Mignon F, Schouman-Claeys E (1999) Failing arteriovenous hemodialysis fistulas: assessment with magnetic resonance angiography. Investig Radiol 34(3):218-224

19. Jin WT, Zhang GF, Liu HC, Zhang H, Li B, Zhu XQ (2015) Non- contrast-enhanced MR angiography for detecting arteriovenous fistula dysfunction in haemodialysis patients. Clin Radiol 70(8):852-857

20. Menegazzo D, Laissy JP, Dürrbach A, Debray MP, Messin B, Delmas V, Mignon F, Schouman-Claeys E (1998) Hemodialysis access fistula creation: preoperative assessment with MR venography and comparison with conventional venography. Radiology 209(3):723-728

21. Bode AS, Planken RN, Merkx MAG, van der Sande FM, Geerts L, Tordoir JHM, Leiner T (2012) Feasibility of non-contrastenhanced magnetic resonance angiography for imaging upper extremity vasculature prior to vascular access creation. Eur J Vasc Endovasc Surg 43(1):88-94

22. Kitagawa H, Kishi T, Saito R, Tomokazu S, Noguchi K, Sunohara N (2008) MR venography using the 3D-MEDIC (multi echo data imaging combination) sequence for lower extremities. Nihon Hoshasen Gijutsu Gakkai Zasshi 64(2):277-285

23. Nayak KS, Nielsen JF, Bernstein MA, Mark1 M, Gatehouse PD, Botnar RM, Saloner D, Lorenz C, Wen H, Hu BS, Epstein FH, Oshinski JN, Raman SV (2015) Cardiovascular magnetic resonance phase contrast imaging. J Cardiovasc Magn Reson 17(1):71

24. Van Bemmelen PS, Kelly P, Blebea J (2005) Improvement in the visualisation of superficial arm veins being evaluated for access and bypass. J Vasc Surg 42(5):957-962

25. Korten E, Toonder IM, Schrama YC, Hop WCJ, van der Ham AC, Wittens CHA (2007) Dialysis fistulae patency and preoperative diameter ultrasound measurements. Eur J Vasc Endovasc Surg 33(4):467-471

26. Silva MB, Hobson RW II, Pappas PJ, Jamil Z, Araki CT, Goldberg MC, Gwertzman G, Padberg FT Jr (1998) A strategy for increasing use of autogenous hemodialysis access procedures: impact of preoperative noninvasive evaluation. J Vasc Surg 27(2):302-308

27. Wong V, Ward R, Taylor J, Selvakumar S, How TV, Bakran A (1996) Factors associated with early failure of arteriovenous fistulae for haemodialysis access. Eur J Vasc Endovasc Surg 12(2):207-213

28. Lauvao LS, Ihnat DM, Goshima KR, Chavez L, Gruessner AC, Mills JL (2009) Vein diameter is the major predictor of fistula maturation. J Vasc Surg 49(6):1499-1504

29. Sigovan M, Rayz V, Gasper W, Alley HF, Owens CD, Saloner D (2013) Vascular remodelling in autogenous arterio-venous fistulas by MRI and CFD. Ann Biomed Eng 41(4):657-668 
30. He Y, Terry CM, Nguyen C, Berceli SA, Shiu YE, Cheung AK (2013) Serial analysis of lumen geometry and hemodynamics in human arteriovenous fistula for hemodialysis using magnetic resonance imaging and computational fluid dynamics. J Biomech 46(1):165-169

31. Pinto C, Hickey R, Carroll TJ, Sato K, Dill K, Omary RA, Kroeker R, Simonetti O, Carr JC (2006) Time-resolved MR angiography with generalized autocalibrating partially parallel acquisition and time-resolved echo-sharing angiographic technique for hemodialysis arteriovenous fistulas and grafts. J Vasc Interv Radiol 17(6):1003-1009 